ORIGINAL ARTICLE

\title{
Nephrolithiasis associated with ceftriaxone therapy: a prospective study in 51 children
}

\author{
Z Avci, A Kokłener, N Uras, F Catal, A Karadag, O Tekin, H Degirmencioglu, E Baskin
}

Arch Dis Child 2004;89:1069-1072. doi: 10.1136/adc.2003.044156

See end of article for authors' affiliations .....................

Correspondence to: Dr Ahmet Karadag, Department of Pediatrics, Faculty of Medicine, Fatih University, Ciftlik Cad No: 57 Bestepe 06510

Ankara, Turkey;

kara_dag@hotmail.com

Revised version received 29 January 2004

Accepted for publication 23 March 2004
Background: Background: Ceftriaxone, a third generation cephalosporin, is widely used for treating infection during childhood. The kidneys eliminate approximately $33-67 \%$ of this agent, and the remainder is eliminated via the biliary system. Ceftriaxone may bind with calcium ions and form insoluble precipitate leading to biliary pseudolithiasis. The aim of this study was to assess whether ceftriaxone associated nephrolithiasis develops by the same mechanism, and whether this condition is dose related.

Methods: The study involved 51 children with various infections. Of these, 24 were hospitalized with severe infection and received $100 \mathrm{mg} / \mathrm{kg} /$ day ceftriaxone divided into two equal intravenous doses. The other 27 patients received a single daily intramuscular injection of $50 \mathrm{mg} / \mathrm{kg} /$ day. Serum and urine parameters were evaluated before and after treatment, and abdominal ultrasonographic examinations were also carried out before and after treatment.

Results: Serum urea, creatinine, and calcium levels were normal in all patients before and after treatment. Post-treatment ultrasound identified nephrolithiasis in four (7.8\%) of the 51 subjects. The stones were all of small size $(2 \mathrm{~mm})$. Comparison of the groups with and without nephrolithiasis revealed no significant differences with respect to age, sex distribution, duration of treatment, or dose/route of administration of ceftriaxone. The renal stones disappeared spontaneously in three of the four cases, but were still present in one patient 7 months after ceftriaxone treatment.

Conclusions: Conclusions: The study showed that children taking a 7 day course of normal or high dose ceftriaxone may develop small sized asymptomatic renal stones. The overall incidence of nephrolithiasis in this study was $7.8 \%$.

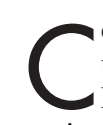

eftriaxone, a third generation cephalosporin, is widely used to treat infections during childhood. Long plasma half life and single dose daily administration are the main advantages of this agent. Ceftriaxone is primarily eliminated via the kidneys (33-67\%), with the remainder eliminated via the biliary system. This drug can bind with calcium ions, producing a precipitate that forms biliary sludge, also known as biliary pseudolithiasis. A number of reports since 1988 have documented biliary pseudolithiasis during ceftriaxone therapy. ${ }^{1-3}$ In addition, nine cases of ceftriaxone induced nephrolithiasis have been described to date, ${ }^{14-10}$ but no prospective investigation of this condition has been carried out. Our aim in this study was to assess for precipitation of ceftriaxone in the renal calyces of a paediatric population and to examine the possible relationship between nephrolithiasis and ceftriaxone dose.

\section{METHODS}

The study involved 51 children who were diagnosed with various types of infection between June 2002 and June 2003. Patients with renal disease, hepatobiliary disease, or any chronic illness, and patients who were already taking nephrotoxic medication were excluded. The study group included 30 girls and 21 boys, and the age range was 1 month to 14 years (mean: 3.1 years; median: 2.5 years). The types of infection were pneumonia $(n=25)$, pyelonephritis $(n=20)$, pneumonia+pyelonephritis $(n=3)$, lymphadenitis $(n=1)$, bacterial meningitis $(n=1)$, and mastoiditis $(n=1)$.

All the patients received the same ceftriaxone preparation (Rocephin). A total of 24 children were hospitalized with severe infection, and this group was treated with intravenous ceftriaxone $100 \mathrm{mg} / \mathrm{kg} /$ day divided into two equal doses. The remaining 27 patients received single daily intramuscular doses of $50 \mathrm{mg} / \mathrm{kg} /$ day ceftriaxone.
For each case, before and after treatment we recorded serum levels of calcium, urea, and creatinine, spot urine levels of calcium and creatinine, and urinalysis findings. We also assessed each child for renal symptoms and complications (colicky abdominal pain, anuria, acute renal failure). For each case of nephrolithiasis, a $24 \mathrm{~h}$ urine sample was collected and excretion levels of calcium, oxalate, citrate, cystine, and uric acid were measured.

In addition to the above tests, all patients underwent abdominal ultrasonography (USG) before and after ceftriaxone therapy. The same $2-5 \mathrm{MHz}$ probe (Sanoline Antares, Siemens, Germany) was used in all cases. Patients who were diagnosed with nephrolithiasis underwent weekly USG exams after treatment to monitor changes in the detected stones.

For each patient, we calculated the urine calcium:creatinine ratio before and after treatment. The means for the two time points were compared to assess the effect of ceftriaxone on urinary calcium excretion.

\section{Statistical analysis}

All the statistical analyses were performed on a personal computer with SPSS (Statistical Package for Social Sciences) version 9.0 for Windows. Mann-Whitney U test was used for analyzing the differences between the mean values of age and duration of treatment of the two groups (with nephrolithiasis and without nephrolithiasis). Wilcoxon test was used to analyze differences between the mean values of urine calcium/creatinine ratios before treatment and after treatment. We have also performed $\chi^{2}$ analysis between the groups with respect to sex and dose. A p value of less than

Abbreviations: USG, ultrasonography 


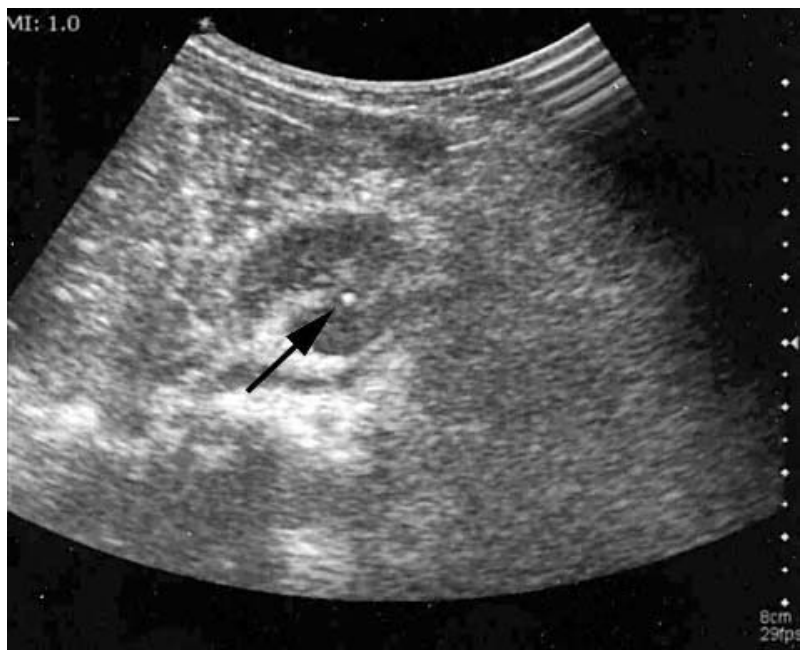

Figure 1 Right kidney sonogram, the white arrow pointing to the calculi in the lower calyx.

0.05 was considered to indicate statistical significance; all tests were two tailed and in the 95\% confidence interval.

\section{RESULTS}

None of the children experienced abdominal pain or developed any renal complications during ceftriaxone therapy. All the patients' serum urea, creatinine, and calcium levels were in the normal range before and after treatment.

None of the children showed abnormalities on abdominal USG before ceftriaxone was administered, but nephrolithiasis was detected in four $(7.8 \%)$ cases after treatment. These patients were three girls and one boy, and their mean (SD) age was 1.1 (0.9) years (range: 0.6-2.5 years).

Fluid restriction was not applied to any of the patients and none of the patients had clinical or laboratory findings of dehydration during the follow up under ceftriaxone treatment.

The mean (SD) duration of treatment in the four patients with nephrolithiasis was 6.75 (0.5) days (range: 6-7 days). The mean (SD) duration of treatment in the 47 patients (20 boys and 27 girls; mean (SD) age: 3.3 (3.2) years; age range: 1 month-14 years) without nephrolithiasis was 7.2 (1) days (range: 5-10 days). Comparisons of these two groups revealed no significant differences with respect to mean age, sex distribution, duration of treatment, or dose/route of ceftriaxone treatment (table 1).

The features of the nephrolithiasis cases are summarized in table 2. One of these patients was a 2.5 year old girl who had received a 7 day course of intramuscular ceftriaxone (50 mg/ $\mathrm{kg} /$ day) for pyelonephritis due to Escherichia coli. Her USG examination on day 7 showed biliary sludge and two renal calculi with a diameter of $2 \mathrm{~mm}$ in the upper pole of the right kidney. The patient exhibited no symptoms. After ceftriaxone was discontinued, serial weekly USG studies showed that the biliary sludge and renal calculi disappeared within 3 weeks.

One of the other patients with nephrolithiasis was a 7 month old boy with learning disabilities but with no feeding problems, who received a 7 day course of intravenous ceftriaxone ( $100 \mathrm{mg} / \mathrm{kg} /$ day) for bacterial pneumonia. We did not recommend any fluid restriction for this patient for pneumonia. In this case, USG on day 7 showed a $2 \mathrm{~mm}$ stone in the lower calyx of the right kidney (fig 1). Follow up with weekly USG revealed no changes in this stone, and it was still present at the patient's most recent USG study done 7 months after treatment.

The other two patients who developed nephrolithiasis both had a single small stone in the lower pole of the left kidney. Serial USG after treatment showed that one of these stones had disappeared by the 3rd week, and the other was gone by the 4 th week.

On ultrasound examination, we observed small (2-3 mm), echogenic calculi within dilated calices in all cases.

The $24 \mathrm{~h}$ urine samples in the four nephrolithiasis cases showed that excretion levels of calcium, oxalate, citrate, cystine, and uric acid were all in the normal range.

There was no significant difference between the mean urine calcium:creatinine ratios for the 51 patients before and after treatment $(0.093 \vee 0.098$, respectively; $\mathrm{p}=1.1)$.

\section{DISCUSSION}

Biliary sludge, or pseudolithiasis, is a known side effect of ceftriaxone treatment. ${ }^{1-3}$ This precipitated material forms when there is a high concentration of ceftriaxone in the biliary system. Normally, the liver eliminates a considerable proportion of ceftriaxone in the form of a soluble salt. However, ceftriaxone is an anion, and, when concentrations of the drug are high, these anions can bind with calcium ions to form insoluble complexes that precipitate out in the biliary system. ${ }^{11-13}$ It appears that stones can form in the same way in the renal collecting system. To date, nine cases of ceftriaxone related nephrolithiasis have been reported, and eight of these patients were children. ${ }^{14-10}$ In five of the nine cases, investigation with infrared spectrophotometry confirmed that the stones had developed due to ceftriaxone therapy.

In our study, post-treatment USG showed that four $(7.8 \%)$ of the 51 children had developed small sized renal calculi while on ceftriaxone. Renal stones are seen on ultrasound examination as echogenic foci with posterior acoustic shadows. However, small stones may fail to demonstrate acoustic shadowing. There is no correlation between stone composition and the sonographic appearance. The clinical history is helpful to distinguish the other echogenic, nonshadowing intraluminal masses including blood clots, pyogenic debris, sloughed papillae in cases of papillary necrosis, fungus balls, and tumours from small stones. ${ }^{14}{ }^{15}$ We supposed that the reaction and subsequently precipitation

Table 1 Features of the 51 cases studied, with patients divided according to ultrasound findings after treatment.

\begin{tabular}{llll}
\hline & \multicolumn{2}{l}{ Ultrasonographic finding } & \\
\cline { 2 - 3 } & Nephrolithiasis $(\mathbf{n}=4)$ & No nephrolithiasis $(\mathbf{n}=\mathbf{4 7})$ & $\mathbf{p}$ \\
\hline Sex (M/F) & $1 / 3$ & $20 / 27$ & 0.634 \\
Age (years), mean (SD); range & $1.1(0.9) ; 0.6-2.5$ & $3.3(3.2) ; 0.08-14$ & 0.146 \\
Duration of treatment (days), mean (SD); range & $6.75(0.5) ; 6-7$ & $7.1(1) ; 5-10$ & 0.151 \\
No. of patients according to Cf dose & 2 & 25 & 1 \\
$\quad 50 \mathrm{mg} / \mathrm{kg} /$ day, im $(\mathrm{n}=27)$ & 2 & 22 & \\
$100 \mathrm{mg} / \mathrm{kg} /$ day, iv $(\mathrm{n}=24)$ & $24)$ & & \\
\hline
\end{tabular}

Cf, Ceftriaxone; im, intramuscular; iv, intravenous. 
Table 2 Clinical features and details of the four nephrolithiasis cases

\begin{tabular}{|c|c|c|c|c|c|c|c|}
\hline No & Sex & Age & Infection type & Cf dose and route & Tx duration (days) & Nephrolithiasis location & Time to disappearance post-Tx \\
\hline 1 & $\mathrm{~F}$ & 2.5 years & Pyelonephritis ( $E$. coli) & $50 \mathrm{mg} / \mathrm{kg} /$ day im & 7 & RK, upper pole & 3 weeks \\
\hline 2 & M & 7 months & Pneumonia & $100 \mathrm{mg} / \mathrm{kg} /$ day iv & 7 & RK, lower calyx & Still present at 7 months \\
\hline 3 & $\mathrm{~F}$ & 7 months & Cervical lymphadenitis & $50 \mathrm{mg} / \mathrm{kg} /$ day im & 5 & LK, lower pole & 4 weeks \\
\hline 4 & $\mathrm{~F}$ & 8 months & Pneumonia & $100 \mathrm{mg} / \mathrm{kg} /$ day iv & 7 & LK, lower pole & 3 weeks \\
\hline
\end{tabular}

of ceftriaxone with calcium ions within renal calices led to the pathogenesis.

It is speculated that metabolic disturbances such as hypercalciuria, hyperuricuria, cystinuria, hyperoxaluria, and hypocitraturia may predispose to the development of nephrolithiasis. ${ }^{16}$ Karliczek et al observed transient hypercalciuria and transient elevation of urinary excretion of oxalate and uric acid in two patients with ceftriaxone related nephrolithiasis. ${ }^{8}$ We observed no such metabolic disturbances in our study. In addition, none of our patients showed a change in urinary excretion of calcium related to ceftriaxone therapy.

Although we applied no fluid restriction and never observed clinical dehydration, mild and clinically undetected dehydration may have existed in our cases due to disease or fever related fluid insufficiency. This phenomenon may have facilitated existing small renal calculi by increasing urinary concentrations of calcium and ceftriaxone in our cases. In previous articles, it has been shown that fluid restriction and dehydration might be risk factors for existing ceftriaxone induced nephrolithiasis. ${ }^{4}$ In another study, nephrotoxic agents have been reported to be a risk factor for large ceftriaxone induced nephrolithiasis. ${ }^{7}$ These articles also confirmed that big stones may cause renal obstruction and impair renal function. None of our cases developed stones large enough to cause obstruction, renal insufficiency, or symptoms of any kind. We believe that the absence of other risk factors such as nephrotoxic medication, liquid restriction, and dehydration, probably helped avoid more serious problems in these cases.

In three cases, as calculi were small and were eliminated spontaneously, we were unable to analyze them using infrared spectrophotometry. In these cases, renal ultrasonographic examinations were initially within normal ranges, and none of the patients had any metabolic disturbance or risk factor leading to stone formation. For these reasons, we strongly consider that ceftriaxone itself was the predisposing factor in the pathophysiology.

In three of our four nephrolithiasis cases, the renal calculus disappeared in a mean time of 3.3 weeks. However, one patient's stone was still present 7 months after treatment. The time for spontaneous elimination of ceftriaxone associated renal stones ranged from 5 days to 3 weeks in previously reported cases. ${ }^{610}$ We observed that the times for spontaneous elimination of calculi in our study were longer than those of previously reported cases. The location of the stone may have been responsible for this as in three cases the calculi were located in the lower pole of the kidney; calculi in this position were reported to be eliminated very late or even never. ${ }^{17}$

Various reports have connected high doses of ceftriaxone with the development of urinary stones. ${ }^{4-8}$ It is also thought that high doses of the drug lead to biliary pseudolithiasis, but this problem also occurs at normal dose levels. ${ }^{2}$ In our study, two of the children who developed nephrolithiasis were receiving $50 \mathrm{mg} / \mathrm{kg} / \mathrm{day}$, and the other two were receiving $100 \mathrm{mg} / \mathrm{kg} / \mathrm{day}$; thus, we found no correlation with dose. Still, high doses may be more likely to lead to the development of large stones with accompanying symptoms. As our cases demonstrate, small sized, asymptomatic stones can form during treatment with normal ceftriaxone doses.

In addition to ceftriaxone dose, longer treatment time can increase a patient's risk for renal complications and nephrolithiasis. In one previously reported case, large obstructive stones were detected on the 4th day of treatment with ceftriaxone $125 \mathrm{mg} / \mathrm{kg} /$ day, ${ }^{6}$ and other patients on a similar dose range developed such stones at 8-10 days of treatment. $^{1479}$ The mean course of treatment in our study was 7 days.

The number of cases in our nephrolithiasis group was very small, so it is impossible to make strong conclusions. However, the male:female ratio in the groups with and without nephrolithiasis was similar, which suggests no sex bias. Interestingly, the mean patient age in the nephrolithiasis group (age (SD) 1.1 (0.9) years) was significantly lower than that of the group without nephrolithiasis (3.3 (3.2) years). This suggests that young age may be a risk factor for the formation of urinary stones during ceftriaxone therapy.

\section{CONCLUSION}

Our results demonstrate that paediatric patients may develop small sized, asymptomatic renal stones during a 7 day course of normal or high dose ceftriaxone therapy. The overall incidence of nephrolithiasis in our study group (27 patients on normal dose, 24 patients on high dose) was $7.8 \%$. It is particularly important to monitor patients on high dose long term ceftriaxone treatment with USG and renal function testing, as these individuals may be at greater risk for large stones and renal damage. This type of screening may help prevent permanent complications in future.

\section{Authors' affiliations}

Z Avci, Department of Pediatrics, Faculty of Medicine, Bașkent University, Ankara, Turkey

A Koktener, Department of Radiology, Faculty of Medicine, Fatih University, Ankara, Turkey

N Uras, F Catal, A Karadag, H Degirmencioglu, Department of Pediatrics, Faculty of Medicine, Fatih University, Ankara, Turkey O Tekin, Department of Family Medicine, Faculty of Medicine, Fatih University, Ankara, Turkey

E Baskin, Pediatric Nephrology Unit, Department of Pediatrics, Faculty of Medicine, Bașkent University, Ankara, Turkey

Conflict of interest: none declared.

\section{REFERENCES}

1 Schaad UB, Wedgwood-Krucko J, Tschaeppeler H. Reversible ceftriaxoneassociated biliary pseudolithiasis in children. Lancet 1988;2:1411-3.

2 Bonnet JP, Abid L, Dabhar A, et al. Early biliary pseudolithiasis during ceftriaxone therapy for acute pyelonephritis in children: a prospective study in 34 children. Eur J Pediatr Surg 2000;10:368-71.

3 Palanduz A, Yalcin I, Tonguc E, et al. Sonographic assessment of ceftriaxoneassociated biliary pseudolithiasis in children. $J$ Clin Ultrasound 2000;28:166-8.

4 Cochat $P$, Cochat $N$, Jouvenet $M$, et al. Ceftriaxone-associated nephrolithiasis. Nephrol Dial Transplant 1990;5:974-6.

5 Feher G, Benczik A, Szabo E, et al. Renal gravel formation inducing renal insufficiency as a side-effect of ceftriaxone. Orv Hetil 1999;140:769-71. 
1072

Asci, Koktener, Urus, et al

6 de Moor RA, Egberts AC, Schroeder CH. Ceftriaxone-associated nephrolithiasis and biliary pseudolithiasis. Eur J Pediatr 1999; 158:975-7.

7 Dulac Y, Bouissou F, Azema C, et al. Anuria caused by urinary lithiasis induced by ceftriaxone in a 6-year-old child. Prese Med 1995;24:916

8 Karliczek SB, Döring S, Vogt S, et al. Ceftriaxone-associated nephrolithiasis. Two case reports. Monatsschr Kinderheilkd 1996;144:702-6.

9 Prince JS, Senac MO Jr. Ceftriaxone-associated nephrolithiasis and biliary pseudolithiasis in a child. Pediatr Radio 2003;33:648-51.

10 Grasberger H, Otto B, Loeschke K. Ceftriaxone-associated nephrolithiasis. Ann Pharmacother 2000;34:1076-7.

11 Park HZ, Lee SP, Shy AL. Ceftriaxone-associated gallbladder sludge. Identification of calcium-ceftriaxone salt as a major component of gallbladder precipitate. Gastroenterology 1991;100:1665-70.
12 Shiffman ML, Keith FB, Moore EW. Pathogenesis of ceftriaxone-associated biliary sludge. In vito studies of calcium-ceftriaxone binding and solubility. Gastroenterology 1990;99:1772-8.

13 Kia Y, Lambert KJ, Schteingard CD, et al. Concentrative biliary secretion of ceftriaxone. Gastroenterology 1990;99:454-65.

14 Rumack CM, Wilson SR, Charboneau JW. Urinary tract. In: Kriegshauser JS, Carroll BA, eds. Diagnostic Ultrasound. St Louis, MO: Mosby, 1991:244-5.

15 King W, Kimme-Smith C, Winder J. Renal stone shadowing: investigation of contributing factor. Radiology 1985;154:191-6.

16 Asplin JR, Favus MJ, Coo FL. Nephrolithiasis. In: Brenner BM, ed. Brenner \& Rector's The Kidney. 6th ed. Philadelphia, PA: WB Saunders, 2000:1774-819.

17 Martin TV, Sosa RE. Shock-wave lithotripsy. In: Walsh PC, Retik AB, Vaughan ED, Ween AJ, eds. Campbell's Urology. Philadelphia, PA: WB Saunders, 1998:2735-52.

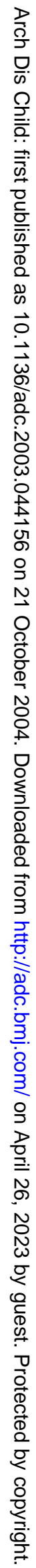

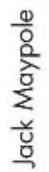

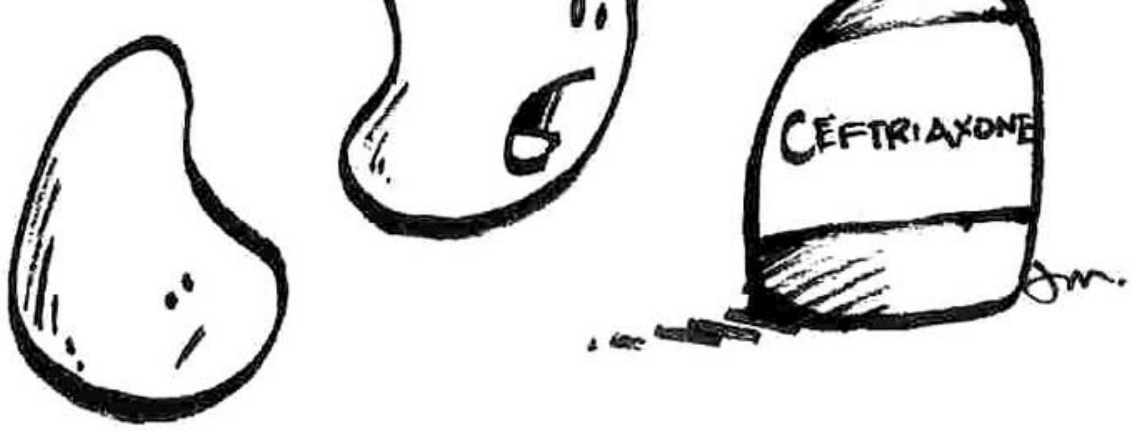

www.archdischild.com 